\title{
Onchocerca volvulus and its Nodule: Examination by Transmission and Scanning Electron Microscopy.
}

\author{
W. J Kozek* and H. Figueroa-Marroquin** \\ *Medical Sciences Campus, University of Puerto Rico, San Juan, PR, 00936-5067 \\ ** Section of Oncocercosis, Ministry of Health and Welfare, Guatemala City, Guatemala
}

Onchocerca volvulus, a viviparous parasitic nematode which lives in subcutaneous nodules in man, is the etiological agent of River Blindness which afflicts more than 30 million persons in many countries of Africa, Central and South America. Its intermediate hosts and transmitters are the flies of the genus Simulium. Scant information available about the ultrastructure of this nematode $[1,2,3]$, and the structure of the nodule that surrounds the worm, prompted us to examine, by transmission and scanning electron microscopy, nodules collected in Guatemala during routine nodulectomy campaign, to elucidate the worm-host interface, ultramorphology of the worm and the cellular architecture of the nodule.

Some of the nodules examined contained young worms and nodules in the state of formation; but most nodules contained mature worms, mostly females, surrounded by well-developed nodules. Much of the stroma of the nodule consists of collagenous fibers that encircle the worms. The spaces between the collagen deposits contain epithelioid cells, fibroblasts, inflammatory cells and blood vessels. The epithelioid cells located near the worm possess extensive cytoplasmic processes which interdigitate with those of the adjacent cells. The host-parasite interface usually consists of a layer of epithelioid cells and a glycocalyx of the superficial layer of the cuticle of the worm (Fig. 1). Accumulations of inflammatory cells have been observed wherever the superfcial glycocalyx appears damaged or is missing. Body wall consists of the cuticle, hypodermal layer and muscle cells. The cuticle is a multilayered structure consisting of an outer fibrous layer, a homogenous layer, internal fibrous layers and the basal layer. Lateral chords, the largest components of the body wall, and much greater in size than are observed in other species of filariae, contain accumulations of bacterial Wolbachia endosymbionts. The surface of the lateral chord that faces the perienteric cavity is fenestrated, the pores representing openings of numerous canaliculi that form a reticular network extending deep into the chords. The canaliculi may serve as conduits for nutrients from the perienteric cavity to the peripheral portions of the nematode. Muscle cells are divided into a contractile and a non-contractile metabolic portion; virus-like particles have been observed within the metabolic portion of several muscle cells near the cytoplasmic extension to the nerve junction. The intestine is a simple tube; intestinal epithelium consists of cuboidal cells resting on thick basal membrane. Oocytes in the ovary and microfilariae developing in the uterus also harbor Wolbachia endosymbionts. The internal membrane of the uterus contains a canalicular system similar to that observed in the lateral chords. The structure of $O$. volvulus suggests that this nematode has made several adaptive changes for its mode of existence. The glyocalyx cuticular surface is unique and may function as an absorptive surface, or it may indicate deposited products of host-parasite 
interaction. The more significant findings include the detection of the Wolbachia endosymbionts and of the virus-like particles within the worms. The presence of Wolbachia in oocyts and developing embryos offer a possible alternate therapeutic strategy to control onchocerciasis by elimination of the endosymbionts. Recent reports suggest that Wolbachial products contribute to the pathological manifestations of onchocerciasis [4,5]; whether the virus-like particles also contribute to the pathological manifestations of this infection remains to be elucidated.

\section{References}

[1] M. Franz, Trop. Med. Parasit. 39 (Suppl. 4) (1988) 359.

[2] G. Strote et al., Parasit. 113 (Pt.1) (1996) 71.

[3] G. Strote et al., Parasit. Res. 83 (1997) 549.

[4] N. W. Brattig at al., Microb. Inf. 2 (2000) 1147.

[5] A. Saint Andre et al., Science 295 (2002) 1892.

[6].Supported, in part, by the RCMI Award RR-03051 from the NCRR, NIH.

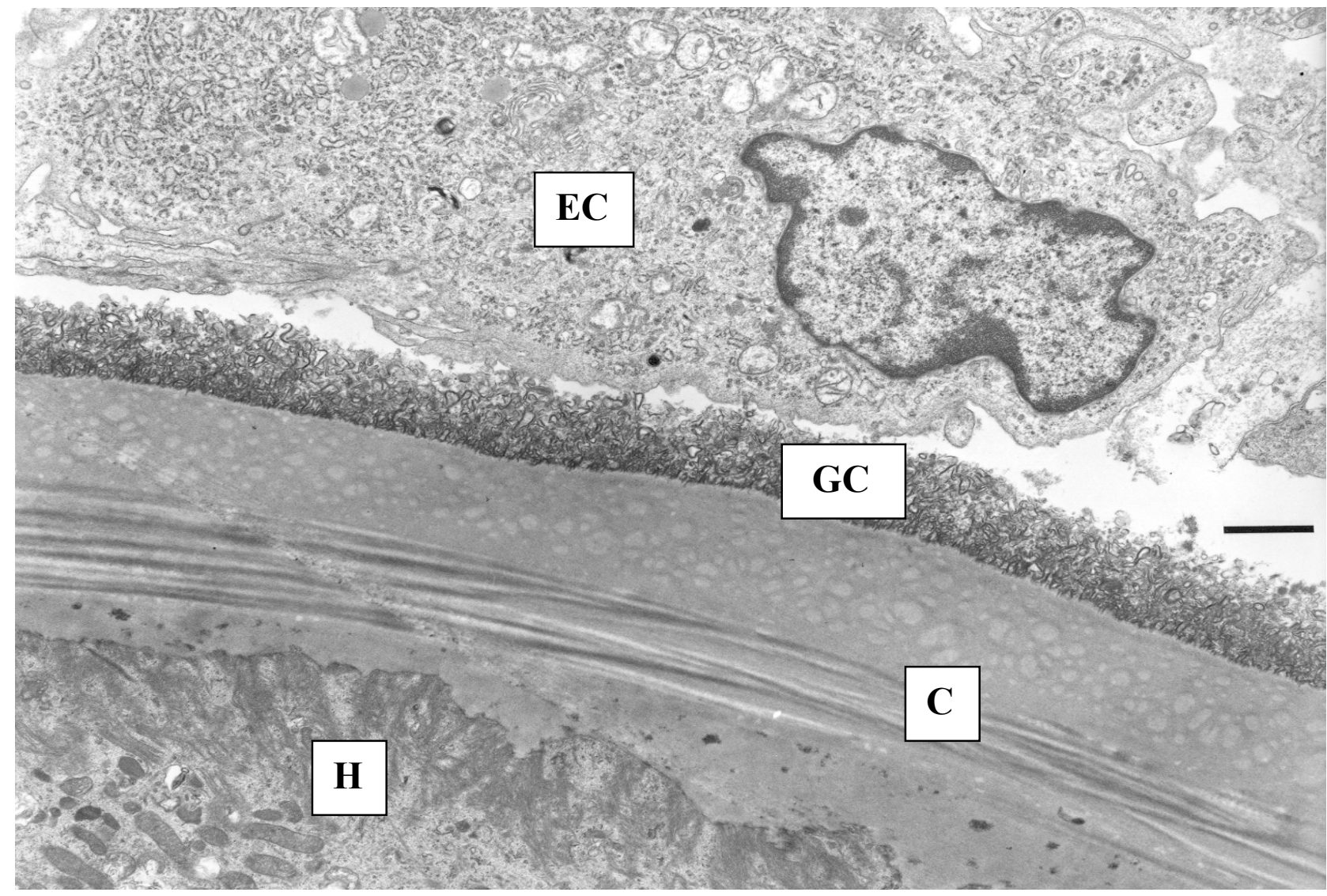

FIG. 1. O. volvulus in situ. Section depicting parasite-host interface. EC - epithelioidal cell, $\mathrm{GC}$ - glycocalyx layer, $\mathrm{C}$ - cuticle, $\mathrm{H}$ - hypodermis. $\mathrm{Bar}=1 \mu \mathrm{m}$. 